\title{
Use of Benzodiazepines and Z-Drugs in Nursing Home Residents with Dementia: Prevalence and Appropriateness
}

\author{
Dirk O.C. Rijksen ${ }^{\mathrm{a}}$, Sytse U. Zuidema ${ }^{\mathrm{b}}$ and Esther C. de Haas ${ }^{\mathrm{b}, *}$ \\ ${ }^{a}$ Carintreggeland, Center for Specialized Geriatric Care, Hengelo, The Netherlands \\ ${ }^{\mathrm{b}}$ Department of General Practice and Elderly Care Medicine, University of Groningen, University Medical \\ Center Groningen, Groningen, the Netherlands
}

Accepted 13 November 2021

Pre-press 2 December 2021

Published 9 December 2021

\begin{abstract}
.
Background: Guidelines worldwide recommend restricted prescription of benzodiazepine receptor agonists (BZRAs), i.e., benzodiazepines and Z-drugs, for the treatment of dementia-associated behavioral and psychological symptoms and insomnia. Objective: To assess the prevalence and appropriateness of BZRA use among nursing home residents with dementia.

Methods: This is a post-hoc analysis of BZRA prescriptions from two intervention studies on psychotropic drug use, conducted from 2016 to 2018. It includes 1,111 residents of dementia special care units from 24 Dutch long-term care organizations. We assessed the prevalence of use of continuous and as-needed BZRA prescriptions and their association with registered symptoms. Continuous BZRA prescriptions were evaluated for appropriateness, i.e., whether indication, dosage, duration, and evaluation accorded with guidelines for the treatment of challenging behavior in dementia and sleep disorders. Results: The prevalence of BZRA use is $39.2 \%$ (95\% CI: 36.3\%-42.0\%): continuous $22.9 \%$; only as-needed $16.3 \%$. Combinations of preferred BZRAs and appropriate indications occur in 19.0\% of continuous anxiolytic prescriptions and $44.8 \%$ of hypnotic prescriptions. Frequently registered inappropriate indications are aggression/agitation for anxiolytics (continuous: 75.7\%; as-needed: $75.2 \%$ ) and nighttime agitation for hypnotics (continuous: $40.3 \%$; as-needed: $26.7 \%$ ). None of the continuous prescriptions with appropriate indications were appropriate for all other items. For most of the prescriptions, duration and time to evaluation exceeded 4 weeks.

Conclusion: BZRA use in nursing home residents with dementia is highly frequent. A large proportion of prescriptions do not follow the guidelines with regard to indication, exceed the recommended duration and are not evaluated in a timely manner. The discrepancy between evidence-based guidelines and daily practice calls for an exploration of factors maintaining inappropriate use.
\end{abstract}

Keywords: Behavioral and psychological symptoms of dementia, benzodiazepines, dementia, inappropriate prescribing, insomnia, nursing homes, Z-drugs

\footnotetext{
${ }^{*}$ Correspondence to: Esther C. de Haas, PhD, Department of General Practice and Elderly Care Medicine, University Medical Center Groningen, PO Box 196, 9700 AD Groningen, the Netherlands. Tel.: +31 5036164 47; E-mail: e.de.haas@umcg.nl.
}

\section{INTRODUCTION}

Benzodiazepine receptor agonists (BZRAs), including benzodiazepines and Z-drugs, are psychotropic drugs that are frequently used in people with dementia. Because of their anxiolytic and sedativehypnotic effects BZRAs are prescribed for the 
treatment of behavioral and psychological symptoms and insomnia. The prevalence of behavioral and psychological symptoms is especially high (approximately 80\%) in long-term care settings [1]. This may contribute to the widespread use of psychotropic drugs among nursing home residents with dementia [2]. According to reports from 2013 the estimated prevalence of BZRA use was around 20\%-25\% [3, 4]. However, more recent data on the frequency and context of use of BZRAs in nursing home residents with dementia are lacking.

Despite the widespread use of BZRAs, there is very limited evidence of their efficacy and safety in people with dementia. Randomized, placebo-controlled research on the efficacy of BZRAs in dementia is limited to one trial that focused on the treatment of acute agitation. A single intramuscular injection of lorazepam led to a clinical response in $72 \%$ of the participants compared to $37 \%$ of the participants in the placebo group [5]. There is a lack of additional randomized, placebo-controlled trials on BZRAs for the treatment of other and less acute behavioral and psychological symptoms as well as insomnia [6-8]. In addition, older persons are known to be especially sensitive to the psychomotor and cognitive side effects of BZRAs, such as drowsiness, dizziness, falls and fractures, cognitive deterioration, and delirium $[9,10]$.

Given the paucity of efficacy data and the risk of side effects, guidelines from around the world recommend restricted prescription of BZRAs to people with dementia [11-16]. In general, psychosocial interventions are encouraged as a first-line approach. The use of BZRAs should be limited to short-term treatment of severe anxiety and primary insomnia (no longer than 2-4 weeks) and to well-circumscribed situations, such as acute crises [9].

In the light of these recommendations, we estimated the prevalence and appropriateness of BZRA use among Dutch nursing home residents with dementia to gain insight into the extent to which prescribing of BZRAs complies with current guidelines.

\section{METHODS}

\section{Study design and participants}

We conducted a post-hoc analysis of BZRA prescription data derived from two intervention studies aimed at reducing inappropriate use of psychotropic drugs in nursing home residents with dementia: Reducing Inappropriate psychotropic Drug use (RID)
[17] and Psychotropic Drug Tool (PDT) [Rasing NL, Janus SIM, Smalbrugge M, Koopmans RTCM, Zuidema SU (2021) Manuscript in preparation].

The RID study is a stepped-wedge cluster randomized controlled trial to study the effects of a tailored intervention and implementation plan for the reduction of inappropriate psychotropic drug prescriptions. The PDT study has a pretest-posttest design and studies the effect of the implementation of a web-based tool for evaluating psychotropic drug prescriptions according to the Dutch guideline for the treatment of challenging behavior in dementia [15]. Both studies included nursing home residents with a chart diagnosis of dementia. Dementia special care units for residents with Korsakov syndrome, acquired brain injury, Down syndrome, or young-onset dementia were excluded. The following baseline information on prescribed psychotropic drugs was collected for both studies: generic agent (Anatomical Therapeutic and Chemical [ATC] classification level 5), registered behavioral and psychological symptoms, dosage, starting date, and date of evaluation (RID study: first date of evaluation; PDT study: date of the most recent evaluation). Both studies were approved by the Medical Ethics Review Board of the University Medical Center Groningen (RID: UMCG 201600160; PDT: UMCG 201800284).

The combined study population consists of 1,111 nursing home residents living at dementia special care units of 24 long-term care organizations throughout the Netherlands (RID study: 576 participants with baseline assessment conducted from July 2016 to July 2017; PDT study: 535 participants evaluated in the period November - December 2018). The mean age is 84.0 years $(\mathrm{SD}=7.7)$ and $814(73.3 \%)$ residents are female.

\section{Assessment of the prevalence of BZRA use}

At the resident level, we assessed whether psychotropic drugs were used and if so, whether these drugs included BZRAs. BZRAs were further classified into the pharmacological subgroups of anxiolytics (ATC3-code N05B) and hypnotics (ATC3-code N05C). We calculated the prevalence of overall use as well as the separate prevalence of continuous and as-needed use, including their $95 \%$ confidence intervals.

\section{Evaluation of the appropriateness of BZRA use}

We assessed the appropriateness of BZRA use at prescription level. We used two Dutch guidelines 
as reference: the multidisciplinary guideline for the treatment of challenging behavior in dementia and the general practitioners' guideline for the treatment of sleep disorders $[15,18]$. Appropriateness is defined as the degree to which BZRA use is in accordance with the recommendations in the guidelines. There are three response categories: fully appropriate, partially appropriate, and inappropriate.

The guideline for the treatment of challenging behavior in dementia recommends restricting BZRA use to the preferred anxiolytics oxazepam (5-30 mg per day) and lorazepam (0.5-1 mg per day) for the treatment of severe anxiety over a maximum period of 4 weeks [15]. It further recommends their use on an as-needed basis in well-circumscribed situations, such as acute crises and dental procedures [15]. For the treatment of primary insomnia, i.e., pre-existent or isolated insomnia, the guideline refers to the guideline for the treatment of sleep disorders. According to this guideline BZRAs should be prescribed only in exceptional cases of insomnia for a maximum of 2 to 4 weeks. Preferred BZRAs are the shortacting hypnotics temazepam (10-20 mg per day), lormetazepam (0.5-1 mg per day), zolpidem ( $5 \mathrm{mg}$ per day), and zopiclon (3.75-7.5 mg per day) [18, 19]. The rationale behind the widespread recommendations to limit use of BZRAs to 2 to 4 weeks is that their efficacy in the treatment of anxiety disorders and insomnia has only been demonstrated for short-term treatment, while tolerance and dependence develop relatively quickly and the risk of side effects persists [9]. Therefore, Dutch guidelines recommend an initial prescription period of 2 weeks, followed by an evaluation and, if necessary, an extension of the prescription to 4 weeks.

We evaluated appropriateness related to registered symptoms, i.e., potential indications, at prescription level for both continuous and as-needed BZRA prescriptions. We used the following non-mutually exclusive categories for behavioral and psychological symptoms of dementia: depressive symptoms, anxiety, psychotic symptoms, agitation/aggression and nighttime agitation (i.e., nocturnal disruption that is considered to be part of the behavioral and psychological symptoms of dementia). In addition, we registered the following other indications for BZRA use: primary insomnia (i.e., pre-existent or isolated insomnia), epilepsy, sedation for a specific procedure, and palliative care. In case of continuous use, we also evaluated the combination of indication and preferred BZRA. An appropriate indication in combination with a preferred BZRA was considered fully appropriate, an appropriate indication in combination with a non-preferred BZRA from the same ATC3-group was considered partially appropriate, and another or no indication was considered inappropriate. Dosage was evaluated according to whether it was within, below or above the recommended dosage or dosage range. The duration of use ( $\leq 2$ weeks, 2-4 weeks, or $>4$ weeks) and the moment of evaluation (RID study: $\leq 2$ weeks, $2-4$ weeks, or $>4$ weeks after starting date; PDT study: $\leq 2$ weeks ago, 2-4 weeks ago, or $>4$ weeks ago) were also evaluated. Two researchers (DR and EdH) scored all items and achieved consensus through discussion. In addition, we calculated the duration of continuous BZRA use from the starting date of the prescription (either at admission or during the stay at the nursing home) up to the date of assessment.

\section{Data analysis}

Descriptive analyses were performed using IBM SPSS Statistics 23 (IBM Corp, Armonk, NY). Since the study populations and observed frequencies are comparable, the data from both studies (RID and PDT) are presented in combination wherever possible. However, information on registered behavioral and psychological symptoms relating to as-needed BZRA use was only available for the PDT study. In addition, the moment of evaluation in the two studies was assessed in different ways (as described in the Methods section).

\section{RESULTS}

\section{Prevalence of BZRA use}

In total, 719 of the 1,111 residents $(64.7 \%$; $95 \% \mathrm{CI}$ : $61.9 \%-67.5 \%)$ use one or more psychotropic drugs and $435(39.2 \%, 95 \%$ CI: $36.3 \%-42.0 \%)$ use a BZRA (Table 1). Continuous BZRA use occurs in 254 residents (22.9\%; 95\% CI: $20.4 \%-25.3 \%)$ : 185 residents (16.7\%; 95\% CI: $14.5 \%-18.8 \%)$ use anxiolytics and 94 (8.5\%; 95\% CI: $6.8 \%-10.1 \%)$ use hypnotics. An additional group of 181 residents $(16.3 \%$; $95 \%$ CI $14.1 \%-18.5 \%$ ) only uses BZRAs on an as-needed basis: $147(13.2 \%$; $95 \%$ CI: $11.2 \%-15.2 \%)$ anxiolytics and 97 (8.7\%; 95\% CI: 7.1\%-10.4\%) hypnotics.

Among the anxiolytic BZRAs, the most frequently prescribed generic agents are oxazepam (continuous use: 67.2\%; as-needed use: $73.2 \%$ ) and lorazepam (continuous use: $30.6 \%$; as-needed use: 
Table 1

Prevalence of BZRA use in nursing home residents with dementia $(n=1,111)$

\begin{tabular}{lccc}
\hline & All BZRAs* & Anxiolytics $^{\dagger}$ & Hypnotics $^{\ddagger}$ \\
\hline Total use & 435 & 332 & 191 \\
& $39.2 \%(36.3-42.0)$ & $29.9 \%(27.2-32.6)$ & $17.2 \%(15.0-19.4)$ \\
Continuous use & 254 & 185 & 94 \\
& $22.9 \%(20.4-25.3)$ & $16.7 \%(14.5-18.8)$ & $8.5 \%(6.8-10.1)$ \\
$\quad$ Continuous use with additional & 138 & 100 & 12 \\
$\quad$ as-needed use & $12.4 \%(10.5-14.4)$ & $9.0 \%(7.3-10.7)$ & $1.1 \%(0.5-1.7)$ \\
As-needed use only & 181 & 147 & 97 \\
& $16.3 \%(14.1-18.5)$ & $13.2 \%(11.2-15.2)$ & $8.7 \%(7.1-10.4)$ \\
\hline
\end{tabular}

*All BZRAs include the anti-epileptic BZRA clonazepam (ATC3 N03AE01), which was used by 7 residents. $\dagger 4$ residents had concurrent prescriptions for 2 anxiolytics: 1 continuous user and 3 as-needed users. $¥ 4$ residents had concurrent prescriptions for 2 hypnotics: 2 continuous users and 2 as-needed users.

Table 2

BZRA prescriptions by generic agent $(n=643)$

\begin{tabular}{lclc}
\hline Anxiolytics (436) & \multicolumn{3}{c}{ Hypnotics (207) } \\
\hline Continuous (186) & Continuous (96) \\
Oxazepam & $125(67.2 \%)$ & Temazepam & $58(60.4 \%)$ \\
Lorazepam & $57(30.6 \%)$ & Midazolam & $16(16.7 \%)$ \\
Alprazolam & $3(1.6 \%)$ & Zopiclon & $12(12.5 \%)$ \\
Diazepam & $1(0.5 \%)$ & Zolpidem & $5(5.2 \%)$ \\
& & Nitrazepam & $4(4.2 \%)$ \\
As-needed (250) & & Lormetazepam & $1(1.0 \%)$ \\
Oxazepam & $183(73.2 \%)$ & As-needed (111) \\
Lorazepam & $59(23.6 \%)$ & Midazolam & $59(53.2 \%)$ \\
Diazepam & $8(3.2 \%)$ & Zolpidem & $5(38.7 \%)$ \\
& & Zopiclon & $3(2.5 \%)$ \\
& & Nitrazepam & $1(0.9 \%)$ \\
\hline
\end{tabular}

$23.6 \%$ ) (Table 2). Temazepam is the most frequently prescribed hypnotic BZRA (continuous use: 60.4\%; as-needed use: $53.2 \%$ ). The most frequently used non-recommended BZRA is the hypnotic midazolam, accounting for $16.7 \%$ of continuous prescriptions and $38.7 \%$ of as-needed prescriptions.

In residents who use BZRAs on a continuous basis as well as on an as-needed basis, the same generic agent is used in 96 out of $100(96 \%)$ anxiolytic prescriptions and 11 out of $12(91.7 \%)$ hypnotic prescriptions (Table 3).

\section{BZRA use and registered symptoms}

Registered symptoms, i.e., potential indications, are lacking for 62 out of $282(22.0 \%)$ continuous BZRA prescriptions and for 11 out of 192 (5.7\%) as-needed BZRA prescriptions (Table 4). Among residents using anxiolytics, anxiety was registered as a symptom for $24.3 \%$ of the continuous prescriptions that included registered symptoms and for $33.1 \%$ of the as-needed prescriptions. Aggression/agitation was registered more frequently as a symptom: for
$75.7 \%$ of the continuous anxiolytic prescriptions and for $75.2 \%$ of the as-needed prescriptions.

Prescriptions of hypnotics are mainly for primary insomnia $(69.4 \%$ of the continuous prescriptions and $48.3 \%$ of the as-needed prescriptions), followed by nighttime agitation $(40.3 \%$ of the continuous prescriptions and $26.7 \%$ of the as-needed prescriptions). Aggression/agitation was registered relatively frequently $(28.3 \%)$ for as-needed hypnotic prescriptions. This symptom is the most frequently registered one for midazolam prescriptions: $50.0 \%$ of continuous prescriptions and $53.8 \%$ of as-needed prescriptions.

\section{Appropriateness of continuous BZRA use: \\ Indication, dosage, duration and evaluation}

A fully appropriate indication, according to the guidelines, is present in 35 out of 186 (19.0\%) continuous anxiolytic prescriptions and 43 out of 96 (44.8\%) continuous hypnotic prescriptions. In these cases, a preferred anxiolytic BZRA was prescribed for anxiety and a preferred hypnotic BZRA was prescribed for primary insomnia. None of these prescriptions are fully appropriate for the complete set of criteria, which also includes dosage, duration, and evaluation apart from indication (Table 5).

Dosage is fully appropriate for $77.1 \%$ of the prescribed anxiolytics and $93.0 \%$ of the prescribed hypnotics. The duration of treatment is least appropriate; none of the anxiolytics and only $16.3 \%$ of the hypnotics were prescribed for a fully appropriate period, i.e., $\leq 2$ weeks. Overall, the median duration of use is 321 days (range 57-1,567) for anxiolytics and 216 days (range 1-1,566) for hypnotics during the stay at the nursing home. The effect of the prescribed BZRA was evaluated within 2 weeks of the starting date for $30.8 \%$ of the anxiolytics and $43.5 \%$ 
Table 3

BZRAs prescribed in individuals with both continuous and as-needed BZRA use

\begin{tabular}{lclc}
\hline \multicolumn{2}{c}{ Anxiolytics: continuous + as-needed (100) } & \multicolumn{1}{c}{ Hypnotics: continuous + as-needed (12) } \\
\hline Oxazepam continuous + as-needed & $72(72.0 \%)$ & Temazepam continuous + as-needed & $7(58.3 \%)$ \\
Lorazepam continuous + as-needed & $24(24.0 \%)$ & Midazolam continuous + as-needed & $4(33.3 \%)$ \\
Lorazepam continuous + oxazepam as-needed & $3(3.0 \%)$ & Temazepam continuous + midazolam as-needed & $1(8.3 \%)$ \\
Diazepam continuous + oxazepam as-needed & $1(1.0 \%)$ & & \\
Same BZRA for continuous + as-needed use & $96(96.0 \%)$ & Same BZRA for continuous + as-needed use & $11(91.7 \%)$ \\
\hline
\end{tabular}

Table 4

Registered symptoms related to prescriptions for continuous and as-needed use of anxiolytic and hypnotic BZRAs*

\begin{tabular}{|c|c|c|c|c|c|c|c|c|c|}
\hline & \multicolumn{9}{|c|}{ Registered symptoms - n (\%) } \\
\hline & \multicolumn{5}{|c|}{ Behavioral and psychological symptoms of dementia } & \multicolumn{3}{|c|}{ Other symptoms } & \multirow[b]{2}{*}{$\begin{array}{l}\text { Palliative } \\
\text { care }\end{array}$} \\
\hline & $\begin{array}{l}\text { Depressive } \\
\text { symptoms }\end{array}$ & Anxiety & $\begin{array}{l}\text { Psychotic } \\
\text { symptoms }\end{array}$ & $\begin{array}{l}\text { Agitation/ } \\
\text { aggression }\end{array}$ & $\begin{array}{l}\text { Nighttime } \\
\text { agitation }\end{array}$ & $\begin{array}{l}\text { Primary } \\
\text { insomnia }\end{array}$ & Epilepsy & $\begin{array}{l}\text { Sedation } \\
\text { (procedure) }\end{array}$ & \\
\hline \multicolumn{10}{|l|}{ Continuous } \\
\hline $\begin{array}{l}\text { Anxiolytics (148) } \\
\text { missing: } 38(20.4 \%)\end{array}$ & $13(8.8 \%)$ & $36(24.3 \%)$ & $2(1.4 \%)$ & $112(75.7 \%)$ & $12(8.1 \%)$ & $15(10.1 \%)$ & & & \\
\hline $\begin{array}{l}\text { Hypnotics (72) } \\
\text { missing: } 24(25.0 \%)\end{array}$ & $1(1.4 \%)$ & $5(6.9 \%)$ & $1(1.4 \%)$ & $10(13.9 \%)$ & $29(40.3 \%)$ & $50(69.4 \%)$ & & & \\
\hline $\begin{array}{l}\text { Midazolam }(14) \\
\text { missing: } 2(12.5 \%)\end{array}$ & $1(7.1 \%)$ & $3(21.4 \%)$ & & $7(50.0 \%)$ & $4(28.6 \%)$ & $5(35.7 \%)$ & & & \\
\hline \multicolumn{10}{|l|}{ As-needed ${ }^{\dagger}$} \\
\hline $\begin{array}{l}\text { Anxiolytics }(121) \\
\text { missing: } 7(5.5 \%)\end{array}$ & $3(2.5 \%)$ & $40(33.1 \%)$ & $4(3.3 \%)$ & $91(75.2 \%)$ & $17(14.0 \%)$ & $7(5.8 \%)$ & $5(4.1 \%)$ & $4(3.3 \%)$ & \\
\hline $\begin{array}{l}\text { Hypnotics }(60) \\
\text { missing: } 4(6.3 \%)\end{array}$ & & $6(10.0 \%)$ & $1(1.7 \%)$ & $17(28.3 \%)$ & $16(26.7 \%)$ & $29(48.3 \%)$ & $3(5.0 \%)$ & $6(10.0 \%)$ & $2(3.3 \%)$ \\
\hline $\begin{array}{l}\text { Midazolam }(26) \\
\text { missing: } 1(3.7 \%)\end{array}$ & & $3(11.5 \%)$ & $1(3.8 \%)$ & $14(53.8 \%)$ & $3(11.5 \%)$ & & $3(11.5 \%)$ & $6(23.1 \%)$ & $2(7.7 \%)$ \\
\hline
\end{tabular}

${ }^{*}$ Registered symptoms are lacking for 62 continuous BZRA prescriptions and for 11 as-needed BZRA prescriptions. The numbers and percentages in the table refer to BZRA prescriptions for which symptoms were registered in the health record. ${ }^{\dagger}$ Information on symptoms in relation to as-needed prescriptions was only available from the PDT study.

Table 5

Appropriateness of dosage, duration, and evaluation of continuous prescriptions of anxiolytic and hypnotic BZRAs which have a fully appropriate indication according to the guidelines

\begin{tabular}{|c|c|c|c|}
\hline Appropriateness & & Anxiolytics $\left(35^{*}\right)$ & Hypnotics $\left(43^{\dagger}\right)$ \\
\hline \multirow[t]{3}{*}{ Dosage } & Within recommended dosage or dosage range & $27(77.1 \%)$ & $40(93.0 \%)$ \\
\hline & Below recommended dosage or dosage range & $0(0.0 \%)$ & $0(0.0 \%)$ \\
\hline & Above recommended dosage or dosage range & $8(22.9 \%)$ & $3(7.0 \%)$ \\
\hline \multirow[t]{3}{*}{ Duration } & $\leq 2$ weeks & $0(0.0 \%)$ & $7(16.3 \%)$ \\
\hline & $\overline{2}-4$ weeks & $0(0.0 \%)$ & $1(2.3 \%)$ \\
\hline & $>4$ weeks & $35(100.0 \%)$ & $35(81.4 \%)$ \\
\hline \multirow[t]{6}{*}{ Evaluation } & $\leq 2$ weeks ago $\ddagger$ & $6^{\ddagger}(27.3 \%)$ & $6^{\ddagger}(30.3 \%)$ \\
\hline & $\leq 2$ weeks after starting date ${ }^{\S}$ & $4^{\S}(30.8 \%)$ & $10^{\S}(43.5 \%)$ \\
\hline & $\overline{2}-4$ weeks ago & $4 \ddagger(18.2 \%)$ & $0^{\ddagger}(0.0 \%)$ \\
\hline & 2-4 weeks after starting date ${ }^{\S}$ & $0^{\S}(0.0 \%)$ & $2^{\S}(8.7 \%)$ \\
\hline & $>4$ weeks ago ${ }^{\ddagger}$ & $12 \ddagger(54.5 \%)$ & $14^{\ddagger}(70.0 \%)$ \\
\hline & $>4$ weeks after starting date ${ }^{\S}$ & $9^{\S}(69.2 \%)$ & $11^{\S}(47.8 \%)$ \\
\hline All items combined & Fully appropriate for all items & $0(0.0 \%)$ & $0(0.0 \%)$ \\
\hline
\end{tabular}

*Indication fully appropriate for 35 out of $186(19.0 \%)$ prescriptions. ${ }^{\dagger}$ indication fully appropriate for 43 out of 96 (44.8\%) prescriptions. ${ }^{\ddagger}$ PDT study. ${ }^{\S}$ RID study. Green: fully appropriate; yellow: partially appropriate; red: inappropriate.

of the hypnotics (RID); and within the last 2 weeks for $27.3 \%$ of the anxiolytics and $30.3 \%$ of the hypnotics (PDT).
Comparable scores for appropriateness are found for the other continuous BZRA prescriptions, which are not fully appropriate with regard to indication 
(Supplementary Table 1 shows the results for all of the continuous BZRA prescriptions).

\section{DISCUSSION}

\section{Main findings}

The estimated prevalence of BZRA users among Dutch nursing home residents with dementia is $39.2 \%$, with $22.9 \%$ of the residents using BZRAs on a continuous basis and an additional $16.3 \%$ using them only on an as-needed basis. The combination of a preferred BZRA with an appropriate indication is found for $19.0 \%$ of the continuous anxiolytic prescriptions and $44.8 \%$ of the hypnotic prescriptions. Inappropriate prescriptions of anxiolytic BZRAs mainly occur in residents exhibiting agitation and aggression, while inappropriate use of hypnotic BZRAs is predominantly found in residents with nighttime agitation and to a lesser degree - in the case of as-needed use-in residents with agitation and aggression. Even in case of appropriate indications, none of the continuous BZRA prescriptions is fully in accordance with the guidelines. This finding can be mainly attributed to long-term use and a lack of timely evaluation.

\section{Prevalence of BZRA use}

Despite the growing restrictiveness of guidelines with regard to prescribing BZRAs, overall BZRA use among nursing home residents with dementia in the two analyzed studies does not appear to be less frequent than BZRA use reported in previous studies. The prevalence of continuous BZRA use in our study (22.9\%) falls within the estimated range of $20 \%-25 \%$ in 2013: in that period a Canadian study reported a prevalence of $17 \%$ for benzodiazepine use, not including the Z-drugs, and a Swedish study reported a prevalence of $29 \%$ for the combined use of anxiolytics and hypnotics, including non-BZRA hypnotics such as melatonin [3, 4]. At the level of pharmacological subgroup, the prevalence of anxiolytic BZRA use $(16.7 \%)$ falls within the range of prevalence values reported for the period 2003-2013 (12\%-21\%) [4, 20-22]. For the complete therapeutic subgroup of hypnotics-sedatives (N05C), which also includes melatonin receptor agonists, the estimated prevalence $(14.0 \%)$ also falls within the range of earlier reported prevalence values $(13 \%-21 \%)$ [4, 20-22].

Few studies have so far reported on the as-needed use of BZRAs in nursing home residents with dementia. In our study, an additional $16.3 \%$ of the residents uses a BZRA only on an as-needed basis; $13.2 \%$ use an anxiolytic BZRA and $8.7 \%$ use a hypnotic BZRA only on an as-needed basis. These values fall within the wide range $(5 \%-31 \%)$ of as-needed use observed among nursing home residents in general [23-25]. A study from the UK specifically reported on asneeded use of benzodiazepines, excluding Z-drugs, in residents with dementia and found a prevalence of $5.4 \%$ [26]. Although the number of benzodiazepine users in this UK study is small $(n=38)$, an estimated $50 \%$ of as-needed prescriptions were actually administered and for some users the frequency of use was almost daily. We were unable to obtain any information on the actual administration of asneeded BZRA prescriptions in our study. As-needed prescriptions may contribute to inappropriate use as a result of unclear instructions [27]. In addition, frequent use of as-needed prescriptions may raise BZRA-related risks, such as falls [28]. As-needed use may especially pose a risk of overexposure to BZRAs in residents who already use BZRAs on a continuous basis (12.4\% of the residents in our study). This risk also applies to residents who have been prescribed both a continuous anxiolytic prescription and a continuous hypnotic prescription $(2.7 \%$ in our study).

\section{Appropriateness of BZRA use}

BZRA prescriptions that do not follow the guidelines are an indicator of BZRA overuse and avoidable BZRA-related risks. In our study, most prescribed BZRAs are not used for the indications mentioned in the relevant guidelines. Anxiety was recorded as a symptom for only $19.0 \%$ of the continuous anxiolytic prescriptions and primary insomnia for only $44.8 \%$ of the hypnotic prescriptions. These figures could have been underestimated, as no description of symptoms could be found in the health records of 20\% and 25\% of the residents with these respective prescriptions.

A striking observation is that agitation/aggression is the most frequently described symptom in residents using an anxiolytic BZRA, despite being considered an inappropriate indication according to the current Dutch guideline. The same issue applies to the use of hypnotic BZRAs in cases of nighttime agitation and daytime agitation/aggression. We also observed that in cases of as-needed prescriptions, the descriptions of symptoms and instructions seldom refer to exceptional, well-circumscribed situations such as emergency situations entailing a significant risk of harm or situations requiring sedation before initiat- 
ing a particular procedure, such as tooth extraction. In the aforementioned cases, the health charts did not list concurrent disorders as alternative indications for BZRA prescriptions, whether or not these prescriptions were off-label.

In addition, we observed an unexpectedly high frequency of off-label use of the hypnotic midazolam, constituting $16.8 \%$ of the continuous hypnotic prescriptions and $38.7 \%$ of the as-needed hypnotic prescriptions. Off-label use of midazolam is especially observed in the context of agitation/aggression, nighttime agitation, and anxiety. Studies on efficacy and safety of midazolam in persons with psychological and behavioral symptoms of dementia are lacking.

None of the continuous BZRA prescriptions with appropriate indications are fully appropriate for all other studied aspects, i.e., dosage, duration of use and timely evaluation. Of the anxiolytic and hypnotic BZRAs, $22.9 \%$ and $7.0 \%$, respectively, are dosed above the recommended range. The duration of use of all anxiolytic BZRAs and $81.4 \%$ of the hypnotic BZRAs is longer than 4 weeks, with median values of 10.5 months for anxiolytics and 7 months for hypnotics. Evaluations of the effects of BZRAs within the first 2 weeks following the starting date took place for only $30.8 \%$ of the anxiolytic prescriptions and $43.5 \%$ of the hypnotic prescriptions. The low adherence to the recommended duration of use is in line with the findings of previous studies on the appropriateness of psychotropic drugs use. In the Dutch PROPER-I study, conducted in 2012, 9.4\% of the prescribed anxiolytics and $31.6 \%$ of the hypnotics were used by nursing home residents with dementia for an appropriate period of time [29]. In addition, the Belgian COME-ON-study, conducted in 2015, found that only $1.7 \%$ of the overall BZRA prescriptions administered to the general nursing home population had a maximum duration of use of 4 weeks [25].

\section{Implications}

There appears to be ample room for improving appropriate prescribing of both anxiolytic and hypnotic BZRAs, according to the current guidelines. Despite a lack of scientific evidence for BZRA use in residents with psychological and behavioral symptoms of dementia and the prevailing notion that their efficacy declines in the long term, BZRAs, especially the anxiolytic ones, are still widely used and their use greatly exceeds the recommended maximum duration of 2-4 weeks.
The questions raised by these findings are as follows: What are the factors underlying the continued prescription of BZRAs beyond the recommendations in the guidelines? What are the prescribers' expectations regarding their efficacy and safety? Are these expectations being influenced by individual experiences? In light of these questions, a randomized placebo-controlled trial on the discontinuation of BZRAs could provide relevant information on the efficacy and safety of BZRAs, especially given that fluctuations in psychological and behavioral symptoms could potentially be erroneously ascribed to the use or discontinuation of BZRAs. Community-based controlled trials in older persons have shown that tapered BZRA withdrawal is feasible and appears to be safe $[30,31]$.

A further question is whether the decision to prescribe and continue BZRAs is a more pragmatic one, driven, for example, by limited time and resources for non-pharmacological interventions, and by experienced pressure to prescribe [32, 33]. Previous studies on deprescribing and reducing inappropriate psychotropic drugs in nursing home residents have entailed interventions like screening tools, team rounds, involvement of residents and relatives, staff education, and multi-strategic and interdisciplinary programs that included medication audits and reviews. Their findings indicate a decrease in use of $21 \%-28 \%$ for BZRAs in general and of $23 \%-40 \%$ specifically for anxiolytics $[25,34,35]$. This raises the question whether there is a lack of implementation of such interventions or whether their implementation has limited success in the long term. Little is known about the considerations of physicians and nurses related to prescribing and use of both continuous and as-needed BZRAs in nursing home residents with dementia. Further exploration of these considerations could contribute to the development of new approaches and tools to support physicians and nurses in appropriate prescribing and administering of BZRAs.

\section{Strengths and limitations}

One of the strengths of this evaluative study, which is based on current guidelines, is the large and representative sample of nursing home residents with dementia. Because medication is administered under the supervision of nursing staff, the prevalence of the evaluated continuous BZRA prescriptions reflects actual BZRA use. In addition to assessing continuous BZRA use, we also assessed BZRA prescriptions on 
an as-needed basis. We conducted an extensive evaluation of BZRA prescriptions in relation to registered psychological and behavioral symptoms and-in the case of continuous BZRA prescriptions - to other aspects of appropriateness.

A limitation related to the study's cross-sectional design is that short-term prescriptions may be underrepresented. Because we conducted a post-hoc analysis of the results of two previous studies, we were unable to estimate a period prevalence, which would have included such short-term prescriptions. In addition, an evaluation of the appropriateness of prescribing is dependent on the quality of reporting in residents' health records. Underreporting may lead to the underestimation of appropriateness. Because the moment of evaluation was assessed in different ways in the two original studies, we were unable to combine the data for this item.

\section{CONCLUSIONS}

Despite a lack of scientific evidence for BZRA use in nursing home residents with dementia and guidelines recommending restraint in prescribing BZRAs, we observe a persistently high prevalence of BZRA use. A large proportion of these BZRA prescriptions, both anxiolytic and hypnotic, do not follow the guidelines with regard to indications, greatly exceed the recommended maximum duration of use and are not evaluated in a timely manner. Further exploration of factors leading to continued BZRA prescribing and use beyond the recommendations in the guidelines, both on continuous and as-needed basis, is required. In the light of our findings, we call for renewed attention to appropriate prescribing of BZRAs and deprescribing in case of inappropriate, long-term use.

\section{ACKNOWLEDGMENTS}

We thank the following researchers for sharing the baseline data from the Reducing Inappropriate psychotropic Drug use (RID) and Psychotropic Drug Tool (PDT) studies: Claudia M. Groot Kormelinck (RID), Charlotte F. van Teunenbroek (RID), Rianne A. Wijbenga (RID) and Naomi L. Rasing (PDT).

\section{FUNDING}

The authors have no funding to report.

\section{CONFLICT OF INTEREST}

The authors have no conflict of interest to report.

\section{SUPPLEMENTARY MATERIAL}

The supplementary material is available in the electronic version of this article: https://dx.doi.org/ 10.3233/ADR-210041.

\section{REFERENCES}

[1] Selbaek G, Engedal K, Bergh S (2013) The prevalence and course of neuropsychiatric symptoms in nursing home patients with dementia: a systematic review. J Am Med Dir Assoc 14, 161-169.

[2] Halvorsen KH, Granas AG, Engeland A, Ruths S (2012) Prescribing quality for older people in Norwegian nursing homes and home nursing services using multidose dispensed drugs. Pharmacoepidemiol Drug Saf 21, 929-936.

[3] Vasudev A, Shariff SZ, Liu K, Burhan AM, Herrmann N, Leonard S, Mamdani M (2015) Trends in psychotropic dispensing among older adults with dementia living in longterm care facilities: 2004-2013. Am J Geriatr Psychiatry 23, 1259-1269.

[4] Gustafsson M, Isaksson U, Karlsson S, Sandman PO, Lövheim H (2016) Behavioral and psychological symptoms and psychotropic drugs among people with cognitive impairment in nursing homes in 2007 and 2013. Eur J Clin Pharmacol 72, 987-994.

[5] Meehan KM, Wang H, David SR, Nisivoccia JR, Jones B, Beasly CM, Feldman PD, Mintzer JE, Beckett LM, Breier A (2002) Comparison of rapidly acting intramuscular olanzapine, lorazepam, and placebo: A double-blind, randomized study in acutely agitated patients with dementia. Neuropsychopharmacology 26, 494-504.

[6] Defrancesco M, Marksteiner J, Fleischhacker WW, Blasko I (2015) Use of benzodiazepines in Alzheimer's disease: A systematic review of literature. Int J Neuropsychopharmacol 18, pyv055.

[7] Tampi RR, Tampi DJ (2014) Efficacy and tolerability of benzodiazepines for the treatment of behavioral and psychological symptoms of dementia: A systematic review of randomized controlled trials. Am J Alzheimers Dis Other Demen 29, 565-574.

[8] McCleery J, Sharpley AL (2020) Pharmacotherapies for sleep disturbances in dementia. Cochrane Database Syst Rev 11, CD009178.

[9] Guina J, Merrill B (2018) Benzodiazepines I: Upping the care on downers: The evidence of risks, benefits and alternatives. J Clin Med 7, 17.

[10] American Geriatrics Society Beers Criteria Update Expert Panel (2019) American Geriatrics Society 2019 updated AGS Beers Criteria for potentially inappropriate medication use in older adults. J Am Geriatr Soc 67, 674-694.

[11] National Collaborating Centre for Mental Health commisioned by the Social Care Institute for Excellence, National Institute for Health and Clinical Excellence (2007) A NICESCIE guideline on supporting people with dementia and their carers in health and social care (National Clinical Practice Guideline Number 42), The British Psychological 
Society and The Royal College of Psychiatrists, Leicester/London.

[12] Canadian Coalition for Seniors' Mental Health (2006) National guidelines for seniors' mental health: The assessment and treatment of mental health issues in long-term care homes (focus on mood and behaviour symptoms), Canadian Coalition for Seniors' Mental Health, Toronto.

[13] APA Work Group on Alzheimer's Disease and other Dementias (2007) American Psychiatric Association practice guideline for the treatment of patients with Alzheimer's disease and other dementias: second edition. Am J Psychiatry 164(12Suppl), 5-56.

[14] National Health and Medical Research Council (2016) Clinical practice guidelines and principles of care for people with dementia, NHMRC Partnership Centre for Dealing with Cognitive and Related Functional Decline in Older People, Sydney.

[15] Zuidema SU, Smalbrugge M, Bil WME, Geelen R, Kok RM, Luijendijk HJ, van der Stelt I, van Strien AM, Vink MT, Vreeken HL (2018) Multidisciplinary Guideline problem behaviour in dementia, Verenso \& NIP, Utrecht.

[16] National Clinical Effectiveness Committee (2019) Appropriate prescribing of psychotropic medication for non-cognitive symptoms in people with dementia (NCEC National Clinical Guideline No. 21), The Department of Health, Dublin.

[17] Groot Kormelinck CM, Van Teunenbroek CF, Kollen BJ, Reitsma M, Gerritsen DL, Smalbrugge M, Zuidema SU (2019) Reducing inappropriate psychotropic drug use in nursing home residents with dementia: Protocol for participatory action research in a stepped-wedge cluster randomized trial. BMC Psychiatry 19, 298.

[18] Gorgels W, Knuistingh Neven A, Lucassen PLBJ, Smelt A, Damen-van Beek Z, Bouma M, Verduijn MM, Van Venrooij M (2014) The Dutch College of General Practitioners' $(N H G)$ guideline Sleep problems and sleeping pills, The Dutch College of General Practitioners, Utrecht.

[19] Dutch National Health Care Institute (Zorginstituut Nederland): Farmacotherapeutisch Kompas. https://farmaco therapeutischkompas.nl, Accessed on March 10, 2021.

[20] Nijk RM, Zuidema SU, Koopmans RTCM (2009) Prevalence and correlates of psychotropic drug use in Dutch nursing-home patients with dementia. Int Psychogeriatr 21, 485-493.

[21] Smeets CHW, Gerritsen DL, Zuidema SU, Teerenstra S, van der Spek K, Smalbrugge M, Koopmans RTCM (2018) Psychotropic drug prescription for nursing home residents with dementia: prevalence and associations with non-residentrelated factors. Aging Ment Health 22, 1239-1246.

[22] Sterke CS, van Beeck EF, van der Velde N, Ziere G, Petrovic M, Looman CWN, van der Cammen TJM (2012) New insights: dose-response relationship between psychotropic drugs and falls: a study in nursing home residents with dementia. J Clin Pharmacol 52, 947-955.

[23] Westbury J, Gee P, Ling T, Kitsos A, Peterson G (2019) More action needed: Psychotropic prescribing in Australian residential aged care. Aust N Z J Psychiatry 53, 136-147.
[24] Picton L, Ilomäki J, Keen CS, Lalic S, Adams B, Clinnick LM, Kirkpatrick CM, Ryan-Atwood Y, Turner JP, Bell JS (2021) Rates of PRN medication administration in Australian residential aged care. J Am Med Dir Assoc 22, 117-123.e1.

[25] Evrard P, Henrard S, Foulon V, Spinewine A (2020) Benzodiazepine use and deprescribing in Belgian nursing homes: Results from the COME-ON study. J Am Geriatr Soc 68 , 2768-2777.

[26] Griffiths AW, Surr CA, Alldred DP, Baker J, Higham R, Spilsbury K, Thompson CA (2019) Pro re nata prescribing and administration for neuropsychiatric symptoms and pain in long-term care residents with dementia and memory problems: a cross-sectional study. Int J Clin Pharm 41, 1314-1322.

[27] Neumann RD, Faris P, Klassen R (2015) Examining trends in the administration of "as needed" medications to inpatients with behavioral and psychological symptoms of dementia. Am J Alzheimers Dis Other Demen 30, 247-256.

[28] Cox CA, van Jaarsveld HJ, Houterman S, van der Stegen JCGH, Wasylewicz ATM, Grouls RJE, van der Linden CMJ (2016) Psychotropic drug prescription and the risk of falls in nursing home residents. J Am Med Dir Assoc 17, 1089-1093.

[29] Van der Spek K, Gerritsen DL, Smalbrugge M, NelissenVrancken MHJMG, Wetzels RB, Smeets CHW, Zuidema SU, Koopmans RTCM (2016) Only 10\% of the psychotropic drug use for neuropsychiatric symptoms in patients with dementia is fully appropriate. The PROPER I-study. Int Psychogeriatr 28, 1589-1595.

[30] Ng BJ, Le Couteur DG, Hilmer SN (2018) Deprescribing benzodiazepines in older patients: impact of interventions targeting physicians, pharmacists, and patients. Drugs Aging 35, 493-521.

[31] Reeve E, Ong M, Wu A, Jansen J, Petrovic M, Gnjidic D (2017) A systematic review of interventions to deprescribe benzodiazepines and other hypnotics among older people. Eur J Clin Pharmacol 73, 927-935.

[32] Wood-Mitchell A, James IA, Waterworth A, Swann A, Ballard C (2008) Factors influencing the prescribing of medications by old age psychiatrists for behavioural and psychological symptoms of dementia: a qualitative study. Age Ageing 37, 547-552.

[33] McCleery J, Fox R (2012) Antipsychotic prescribing in nursing homes. BMJ 344, e1093.

[34] Westbury JL, Gee P, Ling T, Brown DT, Franks KH, Bindoff I, Bindoff A, Peterson GM (2018) RedUSe: reducing antipsychotic and benzodiazepine prescribing in residential aged care facilities. Med J Aust 208, 398-403.

[35] Weeks WB, Mishra MK, Curto D, Petersen CL, Cano P, Hswen Y, Serra SV, Elwyn G, Godfrey MM, Soro PS, Tomás JF (2019) Comparing three methods for reducing psychotropic use in older demented Spanish care home residents. J Am Geriatr Soc 67, 1444-1453. 